\title{
Current patterns of service provision for the psychiatric needs of mentally handicapped people: visiting centres in England and Wales
}

\author{
Gregory O'Brien, Lecturer and Consultant Psychiatrist in Mental Handicap, \\ University Department of Psychiatry, Addenbrooke's Hospital, Cambridge CB2 2QQ
}

In 1988, the Cambridge Health Authority commissioned a working party of clinicians and NHS managers to visit a variety of different treatment services which cater for the psychiatric needs of mentally handicapped adults in England and Wales. This survey - part of an ongoing planning exercise is now complete, new services are being planned in the light of the findings, and the experience may be of general interest to other clinicians and service planners throughout England and Wales.

\section{Outline of the survey}

The survey consisted of five stages. Stage 1 - definition and redefinition of aims; stage 2 -literature search; stage 3-visits to centres; stage 4-data analysis; stage 5-report preparation.

\section{Stage 1: Definition and redefinition of aims}

This initial stage was important. It clarified that the group did not set out either to be concerned solely with the development of a 'treatment unit', nor with a 'community-based team'. From the outset, rather than being committed to either or both of these options, we all agreed that our brief would be to be open-minded, to see what services already exist, and - in the light of the experience of other centres to decide on the most appropriate service model for our local needs.

\section{Stage 2: Literature search}

The literature search was particularly revealing. There seemed to be three types of publication. First of all, there are a number of policy documents, most of which recommend that a community-based service, with some sort of in-patient provision as backup for emergencies and 'special' cases, is required. Secondly, there is a host of publications extolling the virtues of entirely community-based care and crisis intervention teams but, unfortunately, these seem to consist mostly of single case studies. Thirdly, there is a substantial number of accounts of the work of psychiatric treatment in-patient units. These publications, like many of the former policy documents, are generally backed by careful attention to the established prevalence rates of the psychiatric and behavioural problems of mentally handicapped people. A full list of references of the relevant literature survey is available from me.

\section{Stage 3: Visits to other centres}

This part of the survey was characterised by a kind of 'St Elsewhere's' phenomenon. I refer here not to the fictitious American hospital, but to the notion prevalent around the country that somewhere, elsewhere, is a service which is doing all the right things, and this is being done entirely on a community basis. In turn, this is supposed to demonstrate that there is no need for in-patient care in order to best serve the psychiatric needs of disturbed mentally handicapped people. Somehow, this idea seemed to be around in each unit: that such a service existed - always at some distance - and we were repeatedly told that this was somewhere that we really should visit, in order to find the kind of service we should develop. We therefore did follow up each lead given in this way, determined as we were to find such fascinating services. Eventually, we did find one service which had something of this sort - the particular problem we had with respect to this is detailed under Option 2.

It was visiting the various centres which clarified for us that there seemed to be four options open to service planners.

\section{Stage 4: Analysis of data}

The options suggested by the visits to the other centres were:

Option 1. No specialist service for the psychiatric needs of mentally handicapped people. It had been suggested by the literature search that such an approach might have the advantage of avoiding stigmatising, labelling and the fostering of negative and 
dysfunctional expectations among professional staff, clients' families and the clients themselves. We therefore explored this option by visiting centres which had been recommended to the group by authoritative national bodies, as examples of such services. What we in fact found was that such services pay external agencies for hospital admissions. These admissions are generally 'out of county'; in other words, they are more problematic, stigmatising and at a physical distance from the family. We therefore dismissed this option.

Option 2. A community-based specialist service for the psychiatric needs of mentally handicapped people. As the articles supporting this model had indicated, such a service does indeed 'label' certain mentally handicapped people as having psychiatric disorder or behavioural problems. On the other hand, it does deal with the widespread difficulties these clients, families and their carers face. We therefore set out to visit centres where such a service operates. In fact we found only one such service, and this was in Kent. The service in question was exemplary, and certainly dealt adequately with the needs of severely disturbed mentally handicapped people in a natural community setting. However, at the time of visiting, the cost per client of this service was in excess of $£ 100,000$ per year. It was anticipated that this might fall to around $£ 50,000$ per year some time in the future. It was important to note that no other services of this type which we could find in the country purported to cope with such clients: there were other services which had tried, but they had had to have recourse to 'out of county' independent hospital placements or conventional psychiatric treatment units for mentally handicapped people. We therefore felt that this service model was potentially an ideal one, but our health authority did not feel it was financially feasible.

Option 3. A purely hospital-based service. We had in fact been surprised that this had been detailed as one possibility by our hospital managers, in that clinicians had adopted this rather more in Victorian times than at present. In fact, we could not find any such centres in the country and we did not feel it was worth taking this issue further. However, there is a noteworthy development in the private sector, where some residential establishments are beginning to offer lifelong care, from school age upwards. We found this trend very worrying.

Option 4. An integrated community/hospital service. The literature relevant here suggests that such a service can act as an enabling facility to community living, by treating problems in settings in which they arise on the one hand, and by treating more severe problems - when they proved to be untreatable in home settings - in a segregated specialist short-stay unit. There were a number of such centres, but interestingly we found that the nursing staff in such services commonly report two types of deficiency. First of all, it is commonly their impression that a greater degree of intensive professional input into the home setting might be able to deal with the presenting problems, and so obviate the need for admission to hospital in the first place. Interestingly, our visits to other centres where this had been attempted did not in fact substantiate this suggestion: as we indicate under option 1 , we found that those services which 'bend over backwards' to try this approach are also those which more often find their clients in a greater degree of crisis, and need to take precipitate recourse to 'out of county' independent hospital admissions, as opposed to planned admission to a psychiatric treatment unit. The second deficiency which is reported by such units is that of rehabilitation and discharge of problem cases.

\section{Comment}

Not surprisingly, we have chosen option 4 as the only pragmatic service model which might be appropriate for our needs. Within our budgetary constraints, this is the only way we feel that we can offer the comprehensive service our patients require.

To many, particularly to psychiatrists specialising in mental handicap practice, it will seem that we have reinvented the wheel. The idea that both community teams, offering a variety of interventions including long-term support and crisis intervention, should collaborate closely with a specialised in-patient treatment unit geared towards the psychiatric needs of mentally handicapped people, is on first principles quite obvious. However, the importance of the various visits that our survey involved was in clarifying this, not so much for the psychiatrists involved, as for the Health Service planners - both of an administrative and nursing background - and the social services officers and clinical psychologists involved in the survey. For these other professionals, it was most revealing to find that only 'conventional' integrated community and in-patient psychiatric provision does in fact serve the needs of our patients, and does so in the most cost-effective manner. Finally, where such a service model was found to operate in certain centres, we found that staff morale was high, and close interagency multi-disciplinary collaboration was the rule.

\section{Acknowledgements}

We would like to thank the staff in all the various units we visited, who for confidentiality reasons are not named in this report, as had been previously agreed. 